DOI 10.2478/aee-2013-0045

\title{
Decoupling zeros of positive electrical circuits
}

\author{
TADEUSZ KACZOREK \\ Faculty of Electrical Engineering, Białystok University of Technology \\ Wiejska 45D, 15-351 Biatystok \\ e-mail: kaczorek@isep.pw.edu.pl
}

(Received: 25.03.2013, revised: 04.04.2013)

\begin{abstract}
Necessary and sufficient conditions for the reachability and observability of the positive electrical circuits composed of resistors, coils, condensators and voltage sources are established. Definitions of the input-decoupling zeros, output-decoupling zeros and input-output decoupling zeros of the positive electrical circuits are proposed. Some properties of the decoupling zeros of positive electrical circuits are discussed.

Key words: decoupling zeros, positive, continuous-time, linear system, observability, reachability.
\end{abstract}

\section{Introduction}

In positive systems inputs, state variables and outputs take only non-negative values. Examples of positive systems are industrial processes involving chemical reactors, heat exchangers and distillation columns, storage systems, compartmental systems, water and atmospheric pollution models. A variety of models having positive linear behavior can be found in engineering, management science, economics, social sciences, biology and medicine, etc. An overview of state of the art in positive linear theory is given in the monographs [2,3].

The notions of controllability and observability and the decomposition of linear systems have been introduced by Kalman $[9,10]$. Those notions are the basic concepts of the modern control theory $[1,2,4,8,12,16]$. They have been also extended to positive linear systems $[2,3,17]$.

The positivity and reachability to fractional electrical circuits have been investigated in [7]. The decomposition of positive discrete-time linear systems has been addressed in [5]. The notion of the decoupling zeros of standard linear systems have been introduced by Rosenbrock $[11,12]$. The zeros of linear standard system have been addressed in [15] and zeros of positive continuous-time and discrete-time linear systems has been defined in [13, 14]. The decoupling zeros of positive discrete-time linear systems has been introduced in [6].

In this paper the notions of the decoupling zeros will be extended for positive electrical circuits. 
The paper is organized as follows. In Section 2 the basic definitions and theorems concerning reachability and observability of positive electrical circuits are given. The decomposition of the pair (A, B) and (A, C) of positive electrical circuits is addressed in Section 3. The main result of the paper is given in Section 4 where the definitions of the decoupling zeros of positive electrical circuits are proposed. Concluding remarks are given in Section 5.

The following notation will be used: $\mathfrak{R}$ - the set of real numbers, $\mathfrak{R}^{n \times m}$ - the set of $n \times m$ real matrices, $\mathfrak{R}_{+}^{n \times m}$ - the set of $n \times m$ matrices with nonnegative entries and $\mathfrak{R}_{+}^{n}=\mathfrak{R}_{+}^{n \times 1}$, $M_{n}$ - the set of $n \times n$ Metzler matrices (real matrices with nonnegative off-diagonal entries), $I_{n}-$ the $n \times n$ identity matrix.

\section{Reachability and observability of positive electrical circuits}

\subsection{Reachability of positive electrical circuits}

Consider the linear continuous-time electrical circuit described by the equations

$$
\begin{aligned}
& \dot{x}(t)=A x(t)+B u(t) \\
& y(t)=C x(t)+D u(t),
\end{aligned}
$$

where $x(t) \in \mathfrak{R}^{n}, u(t) \in \mathfrak{R}^{m}, y(t) \in \mathfrak{R}^{p}$ are the state, input and output vectors and $A \in \mathfrak{R}^{n \times n}$, $B \in \mathfrak{R}^{n \times m}, C \in \mathfrak{R}^{p \times n}, D \in \mathfrak{R}^{p \times m}$.

Definition 2.1. $[2,3]$ The electrical circuit (2.1) is called (internally) positive if $x(t) \in \mathfrak{R}_{+}^{n}$ and $y(t) \in \mathfrak{R}_{+}^{p}, t \geq 0$ for any $x(0)=x_{0} \in \mathfrak{R}_{+}^{n}$ and every $u(t) \in \mathfrak{R}_{+}^{m}, t \geq 0$.

Theorem 2.1. [2, 3] The electrical circuit (2.1) is positive if and only if

$$
A \in M_{n}, B \in \mathfrak{R}_{+}^{n \times m}, C \in \mathfrak{R}_{+}^{p \times n}, \quad D \in \mathfrak{R}_{+}^{p \times m} .
$$

Definition 2.2. The positive electrical circuit (2.1) (or positive pair $(A, B)$ ) is called reachable at time $t_{f}$ if for any given final state $x_{f} \in \mathfrak{R}_{+}^{n}$ there exists an input $u(t) \in \mathfrak{R}_{+}^{m}, t \in\left[0, t_{f}\right]$ which steers the state of the circuit from the zero state $(x(0)=0)$ to state $x_{f} \in \mathfrak{R}_{+}^{n}$, i.e. $x\left(t_{f}\right)=x_{f}$.

A column $a \in \mathfrak{R}_{+}^{n}$ (row $a^{T} \in \mathfrak{R}_{+}^{n}$ ) is called monomial if only one its entry is positive and the remaining entries are zero. A real matrix $A \in \mathfrak{R}_{+}^{n \times n}$ is called monomial if each its row and each its column contains only one positive entry of its entries and the remaining of its entries are zero.

Theorem 2.2. The positive electrical circuit (2.1) is reachable at time $t \in\left[0, t_{f}\right]$ if and only if the matrix $A \in M_{n}$ is diagonal and the matrix $B \in \mathfrak{R}_{+}^{n \times m}$ is monomial.

Proof. Sufficiency. It is well-known [3] that if $A \in M_{n}$ is diagonal then $e^{A t} \in \mathfrak{R}_{+}^{n \times n}$ is also diagonal and if $B \in \mathfrak{R}_{+}^{n \times m}$ is monomial then $B B^{T} \in \mathfrak{R}_{+}^{n \times n}$ is also monomial. In this case the matrix 


$$
R_{f}=\int_{0}^{t_{f}} e^{A \tau} B B^{T} e^{A^{T} \tau} d \tau \in \mathfrak{R}_{+}^{n \times n}
$$

is also monomial and $R_{f}^{-1} \in \mathfrak{R}_{+}^{n \times n}$. The input

$$
u(t)=B^{T} e^{A^{T}\left(t_{f}-t\right)} R_{f}^{-1} x_{f} \in \mathfrak{R}_{+}^{n \times n} \text { for } t \in\left[0, t_{f}\right]
$$

steers the state $x(t)$ of the circuit from $x(0)=x_{0}=0$ to the state $x\left(t_{f}\right)=x_{f}$ since

$$
\begin{gathered}
x\left(t_{f}\right)=\int_{0}^{t_{f}} e^{A\left(t_{f}-\tau\right)} B u(\tau) d \tau=\int_{0}^{t_{f}} e^{A\left(t_{f}-\tau\right)} B B^{T} e^{A^{T}\left(t_{f}-\tau\right)} d \tau R_{f}^{-1} x_{f} \\
=\int_{0}^{t_{f}} e^{A \tau} B B^{T} e^{A^{T} \tau} d \tau R_{f}^{-1} x_{f}=x_{f} .
\end{gathered}
$$

Necessity. From Cayley-Hamilton theorem we have

$$
e^{A t}=\sum_{k=0}^{n-1} c_{k}(t) A^{k}
$$

where $c_{k}(t), k=0,1, \ldots, n-1$ are some nonzero function of time depending on the matrix $A$. Substitution of (2.6) into

$$
\int_{0}^{t_{f}} e^{A\left(t_{f}-\tau\right)} B u(\tau) d \tau
$$

yields

$$
x_{f}=\left[\begin{array}{llll}
B & A B & \ldots & A^{q-1} B
\end{array}\right]\left[\begin{array}{c}
v_{0}\left(t_{f}\right) \\
v_{1}\left(t_{f}\right) \\
\vdots \\
v_{n-1}\left(t_{f}\right)
\end{array}\right] \text {, }
$$

where

$$
v_{k}\left(t_{f}\right)=\int_{0}^{t_{f}} c_{k}(\tau) u\left(t_{f}-\tau\right) d \tau, \quad k=0,1, \ldots, n-1
$$

For given $x_{f} \in \mathfrak{R}_{+}^{n}$ it is possible to find nonnegative $v_{k}\left(t_{f}\right)$ for $k=0,1, \ldots, n-1$ if and only if the matrix

$$
\left[B A B \ldots A^{q-1} B\right]
$$


has $n$ linearly independent monomial columns and this takes place only if the matrix $[\mathbf{B}, \mathbf{A}]$ contains $n$ linearly independent columns [3]. Note that for the nonnegative $v_{k}\left(t_{f}\right)$, $k=0,1, \ldots, n-1$ it is possible to find a nonnegative input $u(t) \in \mathfrak{R}_{+}^{m}, t \in\left[0, t_{f}\right]$ only if the matrix $B \in \mathfrak{R}_{+}^{n \times m}$ is monomial and the matrix $A \in M_{n}$ is diagonal.

If $m>n$ then the matrix $B \in \mathfrak{R}_{+}^{n \times n}$ should include an $n \times n$ monomial matrix [17].

Now let us consider $n$-mesh electrical circuits with given resistances $R_{k}, k=1, \ldots, q$, inductances $L_{i}, i=1, \ldots, n$ and $m$-mesh source voltages $e_{j}, j=1, \ldots, m$. It is assumed that to each linearly independent mesh belongs only one inductance and one source voltage. In this case the matrix $A \in M_{n}$ and the matrix $B \in \mathfrak{R}_{+}^{n \times n}$ is diagonal and the standard electrical circuit is reachable since $\operatorname{det} B \neq 0$.

Theorem 2.3. The positive $n$-meshes electrical circuit with only one inductance and one source voltage in each linearly independent mesh is reachable if and only if

$$
R_{i j}=0 \text { for } i \neq j,, i, j=1, \ldots, n,
$$

where $R_{i j}$ is the resistance of the branch belonging the $i$-th and $j$-th meshes.

Proof. Note that the matrix $A \in M_{n}$ is also diagonal if and only if the condition (2.11) is met. By Theorem 2.2 the positive electrical circuit is reachable if and only if the condition (2.11) is satisfied since in this case $A \in M_{n}, B \in \mathfrak{R}_{+}^{n \times n}$ are both diagonal.

Example 2.1. Consider the electrical circuit shown in Figure 2.1 with given resistances $R_{1}, R_{2}, R_{3}$, inductances $L_{1}, L_{2}$ and source voltages $e_{1}, e_{2}$.

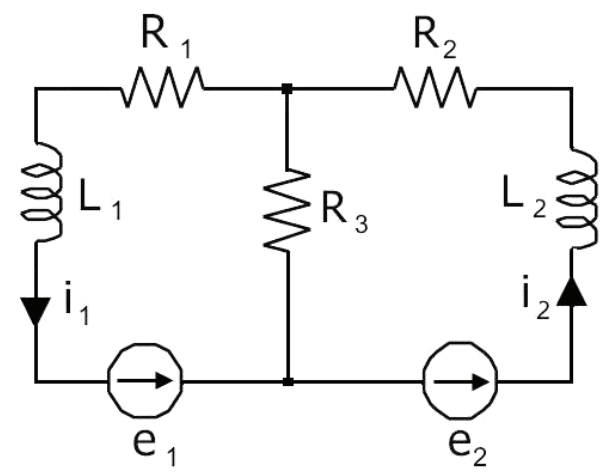

Fig. 2.1. Electrical circuit

Using the Kirchhoff's laws we can write the equations

$$
\begin{aligned}
& e_{1}=R_{3}\left(i_{1}-i_{2}\right)+R_{1} i_{1}+L_{1} \frac{d i_{1}}{d t}, \\
& e_{2}=R_{3}\left(i_{2}-i_{1}\right)+R_{2} i_{2}+L_{2} \frac{d i_{2}}{d t},
\end{aligned}
$$

which can be written in the form 


$$
\frac{d}{d t}\left[\begin{array}{l}
i_{1} \\
i_{2}
\end{array}\right]=\mathbf{A}\left[\begin{array}{l}
i_{1} \\
i_{2}
\end{array}\right]+\mathbf{B}\left[\begin{array}{l}
e_{1} \\
e_{2}
\end{array}\right],
$$

where

$$
\mathbf{A}=\left[\begin{array}{cc}
-\frac{R_{1}+R_{3}}{L_{1}} & \frac{R_{3}}{L_{1}} \\
\frac{R_{3}}{L_{2}} & -\frac{R_{2}+R_{3}}{L_{2}}
\end{array}\right], \quad \mathbf{B}=\left[\begin{array}{cc}
\frac{1}{L_{1}} & 0 \\
0 & \frac{1}{L_{2}}
\end{array}\right]
$$

The electrical circuit is positive since the matrix $\mathbf{A}$ is Metzler and the matrix $\mathbf{B}$ has nonnegative entries. Note that the standard pair (2.13b) is reachable since $\operatorname{det} B \neq 0$.

We shall show that the positive electrical circuit is reachable if $R_{3}=0$. In this case

$$
\mathbf{A}=\left[\begin{array}{cc}
-\frac{R_{1}}{L_{1}} & 0 \\
0 & -\frac{R_{2}}{L_{2}}
\end{array}\right]
$$

and

$$
e^{A \tau}=\left[\begin{array}{cc}
e^{-\frac{R_{1}}{L_{1}} \tau} & 0 \\
0 & e^{-\frac{R_{2}}{L_{2}} \tau}
\end{array}\right]
$$

From (2.3) we obtain

$$
R_{f}=\int_{0}^{t_{f}} e^{A \tau} B B^{T} e^{A^{T} \tau} d \tau=\int_{0}^{t_{f}}\left[\begin{array}{cc}
\frac{1}{L_{1}^{2}} e^{-\frac{2 R_{1}}{L_{1}} \tau} & 0 \\
0 & \frac{1}{L_{2}^{2}} e^{-\frac{2 R_{2}}{L_{2}} \tau}
\end{array}\right] d \tau .
$$

The matrix (2.14) is monomial and by Theorem 2.2 the positive electrical circuit is reachable if $R_{3}=0$.

\subsection{Observability of positive electrical circuits}

Consider a positive electrical circuit described by the state equations

$$
\begin{aligned}
& \dot{x}(t)=A x(t), \\
& y(t)=C x(t),
\end{aligned}
$$

where $x(t) \in \mathfrak{R}_{+}^{n}, y(t) \in \mathfrak{R}_{+}^{p}$ and $A \in M_{n}, C \in \mathfrak{R}_{+}^{p \times n}$. 
Definition 2.3. The positive electrical circuit (2.15) is called observable if knowing the output $y(t) \in \mathfrak{R}_{+}^{p}$ and its derivatives

$$
y^{(k)}(t)=\frac{d^{k} y(t)}{d t^{k}} \in \mathfrak{R}_{+}^{p},
$$

$k=1,2, \ldots, n-1$ for $t \in\left[0, t_{f}\right]$ it is possible to find the initial values $x_{0}=x(0) \in \mathfrak{R}_{+}^{n}$ of $x(t) \in \mathfrak{R}_{+}^{n}$.

Theorem 2.3. The positive electrical circuit (2.15) is observable if and only if the matrix $A \in M_{n}$ is diagonal and the matrix

$$
\left[\begin{array}{c}
C \\
C A \\
\vdots \\
C A^{n-1}
\end{array}\right]
$$

has $n$ linearly independent monomial rows.

Proof. Substituting of the solution

$$
x(t)=e^{A t} x_{0}
$$

of the Equation (2.15a) into (2.15b) yields

$$
y(t)=C e^{A t} x_{0}
$$

From (2.18) we have

$$
\left[\begin{array}{c}
y(t) \\
\dot{y}(t) \\
\vdots \\
y^{(n-1)}(t)
\end{array}\right]=\left[\begin{array}{c}
C \\
C A \\
\vdots \\
C A^{n-1}
\end{array}\right] e^{A t} x_{0} .
$$

It is possible to find from (2.19) $e^{A t} x_{0} \in \mathfrak{R}_{+}^{n}$ if and only if the matrix (2.16) has $n$ linearly independent monomial rows. From the equality $e^{A t} e^{-A t}=I_{n}$ it follows that the matrix $e^{A t} \in \mathfrak{R}_{+}^{n \times n}$ for $A \in M_{n}$ if and only if it is diagonal. Therefore, it is possible to find $x_{0} \in \mathfrak{R}_{+}^{n}$ from the Equation (2.19) if and only if the matrix $A \in M_{n}$ is diagonal and the matrix (2.16) has $n$ linearly independent monomial rows.

Theorem 2.4. The positive electrical circuit (2.15) is observable if the matrix

$$
O_{p}=e^{A^{T} t} C^{T} C e^{A t}
$$

is monomial.

Proof. Premultiplying (2.18) by $e^{A^{T} t} C^{T}$ we obtain 


$$
e^{A^{T} t} C^{T} C e^{A t} x_{0}=e^{A^{T} t} C^{T} y(t)
$$

If the matrix (2.20) is monomial then $O_{p}^{-1}=\left[e^{A^{T} t} C^{T} C e^{A t}\right]^{-1} \in \mathfrak{R}_{+}^{n \times n}$ and from (2.21) we have

$$
x_{0}=\left[e^{A^{T} t} C^{T} C e^{A t}\right]^{-1} e^{A^{T} t} C^{T} y(t) \in \mathfrak{R}_{+}^{n}
$$

since $e^{A^{T} t} C^{T} y(t) \in \mathfrak{R}_{+}^{p}$ for $y(t) \in \mathfrak{R}_{+}^{p}$.

Consider the electrical circuit shown in Figure 2.2 with given conductances $G_{k}, G^{\prime}{ }_{k}, G_{k j}$ $k, j=1, \ldots, n$, capacitances $C_{k}, k=1, \ldots, n$ and source voltages $e_{k}, k=1, \ldots, n$.

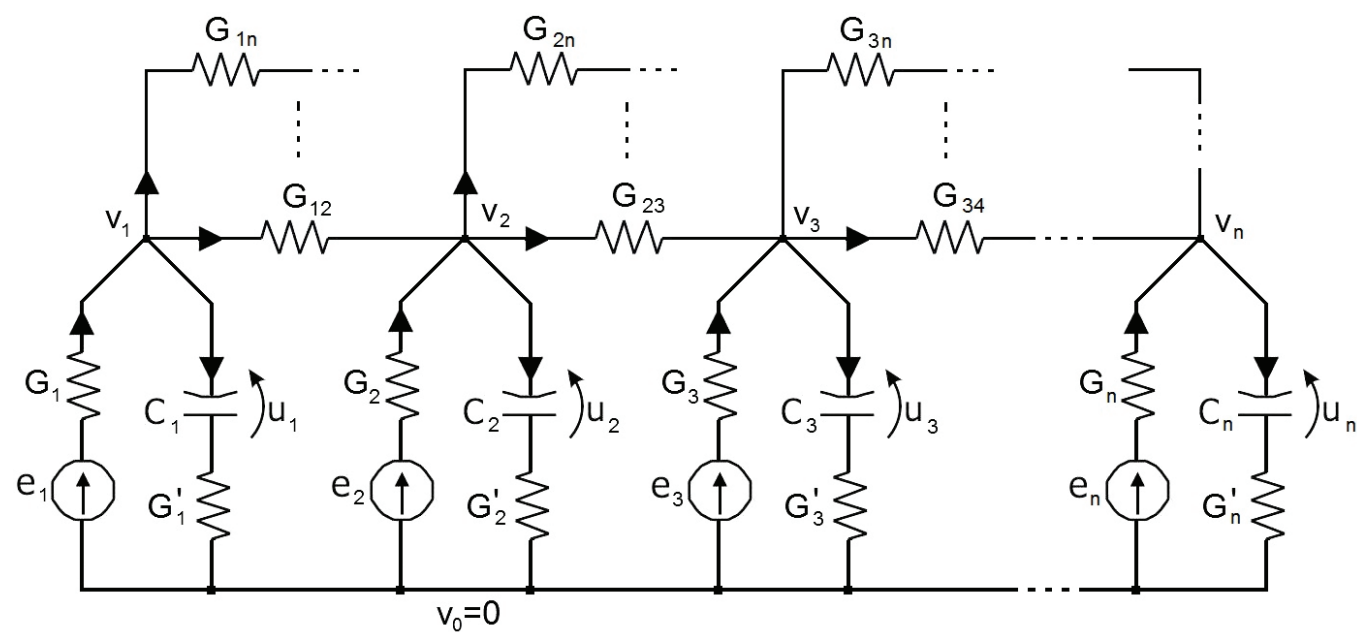

Fig. 2.2. Electrical circuit

Theorem 2.5. The electrical circuit shown in Figure 2.2 is positive for all values of the conductances, capacitances and source voltages.

Proof is given in [7].

Note that the standard electrical circuit shown in Figure 2.2 is reachable for all nonzero values of the conductances and capacitances since $\operatorname{det} B \neq 0$.

Theorem 2.6. The electrical circuit shown in Figure 2.2 is reachable if and only if

$$
G_{k, j}=0 \text { for } k \neq j \text { and } k, j=1, \ldots n \text {. }
$$

Proof. It is easy to see that the matrices $A \in M_{n}$ and $B \in \mathfrak{R}_{+}^{n \times n}$ are both diagonal matrices if and only if the condition (2.23) is satisfied. In this case by Theorem 2.2 the electrical circuit is reachable if and only if the conditions (2.23) are met. 


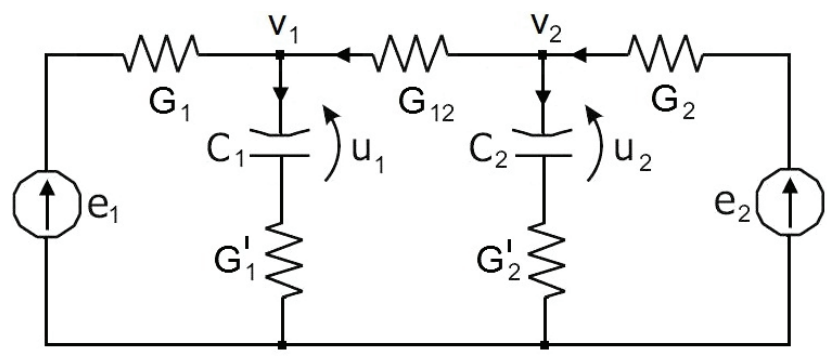

Fig. 2.3. Electrical circuit

Example 2.2. Consider the electrical circuit shown in Figure 2.3 with given conductances $G_{1}, G_{1}^{\prime}, G_{2}, G_{2}^{\prime}, G_{12}$, capacitances $C_{1}, C_{2}$ and source voltages $e_{1}, e_{2}$.

Using the Kirchhoff's laws we can write the equations

$$
\frac{d}{d t}\left[\begin{array}{l}
u_{1} \\
u_{2}
\end{array}\right]=\left[\begin{array}{cc}
\frac{G_{1}^{\prime}}{C_{1}} & 0 \\
0 & \frac{G_{2}^{\prime}}{C_{2}}
\end{array}\right]\left[\begin{array}{l}
v_{1} \\
v_{2}
\end{array}\right]-\left[\begin{array}{cc}
\frac{G_{1}^{\prime}}{C_{1}} & 0 \\
0 & \frac{G_{2}^{\prime}}{C_{2}}
\end{array}\right]\left[\begin{array}{l}
u_{1} \\
u_{2}
\end{array}\right]
$$

and

$$
\left[\begin{array}{cc}
-G_{11} & G_{12} \\
G_{12} & -G_{22}
\end{array}\right]\left[\begin{array}{l}
v_{1} \\
v_{2}
\end{array}\right]=-\left[\begin{array}{cc}
G_{1}^{\prime} & 0 \\
0 & G^{\prime}
\end{array}\right]\left[\begin{array}{l}
u_{1} \\
u_{2}
\end{array}\right]-\left[\begin{array}{cc}
G_{1} & 0 \\
0 & G_{2}
\end{array}\right]\left[\begin{array}{l}
e_{1} \\
e_{2}
\end{array}\right],
$$

where

$$
G_{11}=G_{1}+G_{1}^{\prime}+G_{12}, G_{22}=G_{2}+G_{2}^{\prime}+G_{12} .
$$

Taking into account that the matrix

$$
\left[\begin{array}{cc}
-G_{11} & G_{12} \\
G_{12} & -G_{22}
\end{array}\right]
$$

is nonsingular and

$$
-\left[\begin{array}{cc}
-G_{11} & G_{12} \\
G_{12} & -G_{22}
\end{array}\right]^{-1} \in \mathfrak{R}_{+}^{2 \times 2}
$$

from (2.25) we obtain

$$
\left[\begin{array}{l}
v_{1} \\
v_{2}
\end{array}\right]=-\left[\begin{array}{cc}
-G_{11} & G_{12} \\
G_{12} & -G_{22}
\end{array}\right]^{-1}\left\{\left[\begin{array}{cc}
G_{1}^{\prime} & 0 \\
0 & G_{2}^{\prime}
\end{array}\right]\left[\begin{array}{l}
u_{1} \\
u_{2}
\end{array}\right]+\left[\begin{array}{cc}
G_{1} & 0 \\
0 & G_{2}
\end{array}\right]\left[\begin{array}{l}
e_{1} \\
e_{2}
\end{array}\right]\right\} .
$$

Substitution of (2.28) into (2.24) yields

$$
\frac{d}{d t}\left[\begin{array}{l}
u_{1} \\
u_{2}
\end{array}\right]=A\left[\begin{array}{l}
u_{1} \\
u_{2}
\end{array}\right]+B\left[\begin{array}{l}
e_{1} \\
e_{2}
\end{array}\right],
$$


where

$$
\begin{gathered}
A=-\left[\begin{array}{cc}
\frac{G_{1}^{\prime}}{C_{1}} & 0 \\
0 & \frac{G_{2}^{\prime}}{C_{2}}
\end{array}\right]\left[\begin{array}{cc}
-G_{11} & G_{12} \\
G_{12} & -G_{22}
\end{array}\right]^{-1}\left[\begin{array}{cc}
G_{1}^{\prime} & 0 \\
0 & G_{2}^{\prime}
\end{array}\right]-\left[\begin{array}{cc}
\frac{G_{1}^{\prime}}{C_{1}} & 0 \\
0 & \frac{G_{2}^{\prime}}{C_{2}}
\end{array}\right] \in M_{2}, \\
B=-\left[\begin{array}{cc}
\frac{G_{1}^{\prime}}{C_{1}} & 0 \\
0 & \frac{G_{2}^{\prime}}{C_{2}}
\end{array}\right]\left[\begin{array}{cc}
-G_{11} & G_{12} \\
G_{12} & -G_{22}
\end{array}\right]^{-1}\left[\begin{array}{cc}
G_{1} & 0 \\
0 & G_{2}
\end{array}\right] \in \mathfrak{R}_{+}^{2 \times 2} .
\end{gathered}
$$

From (2.30) it follows that $A$ is Metzler matrix and the matrix $B$ has nonnegative entries. Therefore, the electrical circuit is positive for all values of the conductances and capacitances.

\section{Decomposition of the pairs $(A, B)$ and $(A, C)$}

\subsection{Decomposition of the pair $(A, B)$}

Consider the pair $(A, B)$ with $A$ being diagonal

$$
A=\operatorname{diag}\left[a_{11}, a_{22}, \ldots, a_{n, n}\right] \in M_{n}
$$

and the matrix $B$ with $m$ linearly independent monomial columns $B_{1}, B_{2}, \ldots, B_{m}$

$$
B=\left[\begin{array}{llll}
B_{1} & B_{2} & \ldots & B_{m}
\end{array}\right] .
$$

By Theorem 2.2 the pair (3.1) is unreachable if $m<n$.

It will be shown that in this case the pair can be decomposed into the reachable pair $\left(\bar{A}_{1}, \bar{B}_{1}\right)$ and unreachable pair $\left(\bar{A}_{2}, \bar{B}_{2}=0\right)$.

Theorem 3.1. For the unreachable pair (3.1) $(m<n)$ there exists a monomial matrix $P \in \Re_{+}^{n \times n}$ such that

$$
\bar{A}=P A P^{-1}=\left[\begin{array}{cc}
\bar{A}_{1} & 0 \\
0 & \bar{A}_{2}
\end{array}\right], \bar{B}=P B=\left[\begin{array}{c}
\bar{B}_{1} \\
0
\end{array}\right],
$$

where $\bar{A}_{1}=\operatorname{diag}\left[\bar{a}_{11}, \bar{a}_{22}, \ldots, \bar{a}_{n_{1}, n_{1}}\right] \in M_{n_{1}}, \quad \bar{A}_{2}=\operatorname{diag}\left[\bar{a}_{n_{1}+1, n_{1}+1}, \ldots, \bar{a}_{n, n}\right] \in M_{n_{2}}, \quad \bar{B}_{1} \in \mathfrak{R}_{+}^{n_{1} \times m}$, $n=n_{1}+n_{2}$, the pair $\left(\bar{A}_{1}, \bar{B}_{1}\right)$ is reachable and the pair $\left(\bar{A}_{2}, \bar{B}_{2}=0\right)$ is unreachable.

Proof. Performing on the matrix $B$ the following elementary row operations:

1) interchange the $i$-th and $j$-th rows, denoted by $L[i, j]$,

2) multiplication of $i$-th rows by positive number $c$, denoted by $L[i \times c]$, we may reduced the matrix $B$ to the form

$$
\left[\begin{array}{c}
\bar{B}_{1} \\
0
\end{array}\right]
$$


where $\bar{B}_{1} \in \Re_{+}^{n_{1} \times m}$ is monomial with positive entries equal to 1 . Performing the same elementary row operations on the identity matrix $I_{n}$ we obtain the desired monomial matrix $P$. It is well-known [3] that $P^{-1} \in \mathfrak{R}_{+}^{n \times n}$ and for diagonal matrix $A$ we have

$$
\bar{A}=P A P^{-1}=\left[\begin{array}{cc}
\bar{A}_{1} & 0 \\
0 & \bar{A}_{2}
\end{array}\right] .
$$

Example 3.1. Consider the electrical circuit shown in Figure 3.1 with given resistances $R_{1}, R_{2}, R_{3}$, inductances $L_{1}, L_{2}, L_{3}$ and source voltages $e_{1}, e_{3}$.

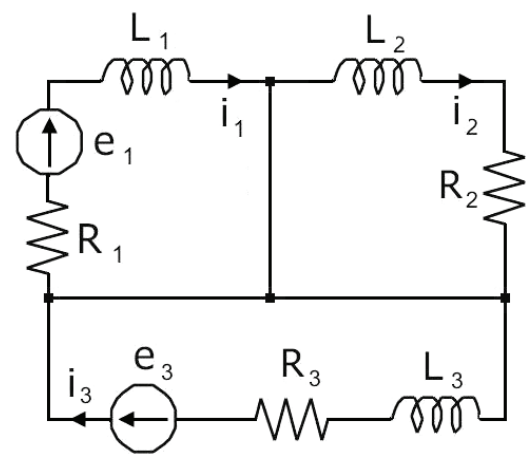

Fig. 3.1. Electrical circuit

Using the Kirchhoff's laws we can write the equations

$$
\begin{aligned}
& L_{1} \frac{d i_{1}}{d t}=-R_{1} i_{1}+e_{1} \\
& L_{2} \frac{d i_{2}}{d t}=-R_{2} i_{2} \\
& L_{3} \frac{d i_{3}}{d t}=-R_{3} i_{3}+e_{3},
\end{aligned}
$$

which can be written in the form

$$
\frac{d}{d t}\left[\begin{array}{l}
i_{1} \\
i_{2} \\
i_{3}
\end{array}\right]=A\left[\begin{array}{l}
i_{1} \\
i_{2} \\
i_{3}
\end{array}\right]+B\left[\begin{array}{l}
e_{1} \\
e_{2}
\end{array}\right],
$$

where

$$
A=\left[\begin{array}{ccc}
-\frac{R_{1}}{L_{1}} & 0 & 0 \\
0 & -\frac{R_{2}}{L_{2}} & 0 \\
0 & 0 & -\frac{R_{3}}{L_{3}}
\end{array}\right], B=\left[\begin{array}{cc}
\frac{1}{L_{1}} & 0 \\
0 & 0 \\
0 & \frac{1}{L_{3}}
\end{array}\right] .
$$


By Theorem 2.2 the positive electrical circuit (or the pair (3.4b)) is unreachable since $n=3$ $<m=2$.

The unreachable pair (3.4b) can be decomposed into reachable pair $\left(\bar{A}_{1}, \bar{B}_{1}\right)$ and unreachable pair $\left(\bar{A}_{2}, \bar{B}_{2}=0\right)$.

In this case the monomial matrix $P$ has the form

$$
P=\left[\begin{array}{lll}
1 & 0 & 0 \\
0 & 0 & 1 \\
0 & 1 & 0
\end{array}\right]
$$

and we obtain

$$
\begin{aligned}
& \bar{B}=P B=\left[\begin{array}{lll}
1 & 0 & 0 \\
0 & 0 & 1 \\
0 & 1 & 0
\end{array}\right]\left[\begin{array}{cc}
\frac{1}{L_{1}} & 0 \\
0 & 0 \\
0 & \frac{1}{L_{3}}
\end{array}\right]=\left[\begin{array}{cc}
\frac{1}{L_{1}} & 0 \\
0 & \frac{1}{L_{3}} \\
0 & 0
\end{array}\right]=\left[\begin{array}{c}
\bar{B}_{1} \\
0
\end{array}\right], \bar{B}_{1}=\left[\begin{array}{cc}
\frac{1}{L_{1}} & 0 \\
0 & \frac{1}{L_{3}}
\end{array}\right], \\
& \bar{A}=P A P^{-1}=\left[\begin{array}{lll}
1 & 0 & 0 \\
0 & 0 & 1 \\
0 & 1 & 0
\end{array}\right]\left[\begin{array}{ccc}
-\frac{R_{1}}{L_{1}} & 0 & 0 \\
0 & -\frac{R_{2}}{L_{2}} & 0 \\
0 & 0 & -\frac{R_{3}}{L_{3}}
\end{array}\right]\left[\begin{array}{lll}
1 & 0 & 0 \\
0 & 0 & 1 \\
0 & 1 & 0
\end{array}\right]=\left[\begin{array}{ccc}
-\frac{R_{1}}{L_{1}} & 0 & 0 \\
0 & -\frac{R_{3}}{L_{3}} & 0 \\
0 & 0 & -\frac{R_{2}}{L_{2}}
\end{array}\right]=\left[\begin{array}{cc}
\bar{A}_{1} & 0 \\
0 & \bar{A}_{2}
\end{array}\right]
\end{aligned}
$$

and

$$
\bar{A}_{1}=\left[\begin{array}{cc}
-\frac{R_{1}}{L_{1}} & 0 \\
0 & -\frac{R_{3}}{L_{3}}
\end{array}\right], \quad \bar{A}_{2}=\left[-\frac{R_{2}}{L_{2}}\right]
$$

The reachable pair $\left(\bar{A}_{1}, \bar{B}_{1}\right)$ is reachable and the pair $\left(\bar{A}_{2}, \bar{B}_{2}=0\right)$ is unreachable.

\subsection{Decomposition of the pair $(A, C)$}

Let the observability matrix

$$
O_{n}=\left[\begin{array}{c}
C \\
C A \\
\vdots \\
C A^{n-1}
\end{array}\right] \in \mathfrak{R}_{+}^{p n \times n}
$$

of the positive unobservable electrical circuit has $n_{1}<n$ linearly independent monomial rows.

If the conditions 


$$
Q_{k} A Q_{j}^{T}=0 \text { for } k=1,2, \ldots, \hat{n}_{1} \text { and } j=\hat{n}_{1}+1, \ldots, n
$$

are satisfied then there exists the monomial matrix $[5,6]$

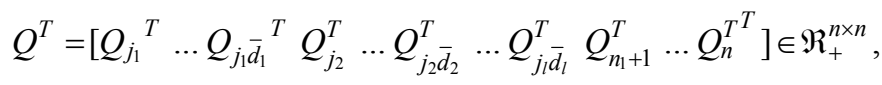

where

$$
Q_{j_{1}}=C_{j_{1}}, \ldots, Q_{j_{1} \bar{d}_{1}}=C_{j_{1}} A^{\bar{d}_{1}-1}, Q_{j_{2}}=C_{j_{2}}, \ldots, Q_{j_{2} \bar{d}_{2}}=C_{j_{2}} A^{\bar{d}_{2}-1}, \ldots, Q_{j_{l} \bar{d}_{l}}=C_{j_{l}} A^{\bar{d}_{l}-1}
$$

and $\bar{d}_{j}, j=1, \ldots, l$ are some natural numbers.

Theorem 3.2. Let the positive electrical circuit (2.15) be unobservable and let there exist the monomial matrix (3.10). Then the pair $(A, C)$ of the electrical circuit can be reduced by the use of the matrix (3.10) to the form

$$
\begin{gathered}
\hat{A}=Q A Q^{-1}=\left[\begin{array}{cc}
\hat{A}_{1} & 0 \\
\hat{A}_{21} & \hat{A}_{2}
\end{array}\right], \hat{C}=C Q^{-1}=\left[\begin{array}{ll}
\hat{C}_{1} & 0
\end{array}\right] \\
\hat{A}_{1} \in \mathfrak{R}_{+}^{n_{1} \times n_{1}}, \quad \hat{A}_{2} \in \mathfrak{R}_{+}^{n_{2} \times n_{2}}, \quad\left(n_{2}=n-n_{1}\right) \quad \hat{A}_{21} \in \mathfrak{R}_{+}^{n_{2} \times n_{1}}, \quad \hat{C}_{1} \in \mathfrak{R}_{+}^{p \times n_{1}},
\end{gathered}
$$

where the pair $\left(\hat{A}_{1}, \hat{C}_{1}\right)$ is observable and the pair $\left(\hat{A}_{2}, \hat{C}_{2}=0\right)$ is unobservable. Proof is given in [5].

Example 3.2. Consider the positive electrical circuit shown in Figure 3.1 described by the state equation (3.4a) with $A$ given by (3.4b). As the output $y(t)$ we assume

$$
y(t)=R_{3} i_{3}=\left[\begin{array}{lll}
0 & 0 & R_{3}
\end{array}\right]\left[\begin{array}{l}
i_{1} \\
i_{2} \\
i_{3}
\end{array}\right], \quad C=\left[\begin{array}{lll}
0 & 0 & R_{3}
\end{array}\right] .
$$

In this case the observability matrix

$$
O_{n}=\left[\begin{array}{c}
C \\
C A \\
C A^{2}
\end{array}\right]=\left[\begin{array}{ccc}
0 & 0 & R_{3} \\
0 & 0 & -\frac{R_{3}^{2}}{L_{3}} \\
0 & 0 & \frac{R_{3}^{3}}{L_{3}^{2}}
\end{array}\right]
$$

has only one linearly independent monomial row $Q_{1}=C$, i.e. $n_{1}=1$ and the conditions (3.9) 
are satisfied for $Q_{2}=\left[\begin{array}{lll}1 & 0 & 0\end{array}\right]$ and $Q_{3}=\left[\begin{array}{lll}0 & 1 & 0\end{array}\right]$ since $Q_{1} A Q_{j}^{T}=0$ for $j=2,3$. The matrix (3.10) has the form

$$
Q=\left[\begin{array}{l}
Q_{1} \\
Q_{2} \\
Q_{3}
\end{array}\right]=\left[\begin{array}{lll}
0 & 0 & R_{3} \\
1 & 0 & 0 \\
0 & 1 & 0
\end{array}\right]
$$

Using (3.11) we obtain

$$
\begin{aligned}
& \hat{A}=Q A Q^{-1}=\left[\begin{array}{ccc}
0 & 0 & R_{3} \\
1 & 0 & 0 \\
0 & 1 & 0
\end{array}\right]\left[\begin{array}{ccc}
-\frac{R_{1}}{L_{1}} & 0 & 0 \\
0 & -\frac{R_{2}}{L_{2}} & 0 \\
0 & 0 & -\frac{R_{3}}{L_{3}}
\end{array}\right]\left[\begin{array}{ccc}
0 & 1 & 0 \\
0 & 0 & 1 \\
\frac{1}{R_{3}} & 0 & 0
\end{array}\right]=\left[\begin{array}{ccc}
-\frac{R_{3}}{L_{3}} & 0 & 0 \\
0 & -\frac{R_{1}}{L_{1}} & 0 \\
0 & 0 & -\frac{R_{2}}{L_{2}}
\end{array}\right]=\left[\begin{array}{cc}
\hat{A}_{1} & 0 \\
0 & \hat{A}_{2}
\end{array}\right], \\
& \hat{C}=C Q^{-1}=\left[\begin{array}{lll}
0 & 0 & R_{3}
\end{array}\right]\left[\begin{array}{ccc}
0 & 1 & 0 \\
0 & 0 & 1 \\
\frac{1}{R_{3}} & 0 & 0
\end{array}\right]=\left[\begin{array}{lll}
1 & 0 & 0
\end{array}\right]=\left[\begin{array}{ll}
\hat{C}_{1} & 0
\end{array}\right] \text {, }
\end{aligned}
$$

where

$$
\hat{A}_{1}=\left[-\frac{R_{3}}{L_{3}}\right], \quad \hat{A}_{2}=\left[\begin{array}{cc}
-\frac{R_{1}}{L_{1}} & 0 \\
0 & -\frac{R_{2}}{L_{2}}
\end{array}\right], \quad \hat{C}_{1}=[1] .
$$

The pair $\left(\hat{A}_{1}, \hat{C}_{1}\right)$ is observable and the pair $\left(\hat{A}_{2}, \hat{C}_{2}=0\right)$ is unobservable.

\section{Decoupling zeros of the positive electrical circuits}

It is well-known [12] that for standard linear systems the input-decupling zeros are the eigenvalues of the matrix $\bar{A}_{2}$ of the unreachable (uncontrollable) part $\left(\bar{A}_{2}, \bar{B}_{2}=0\right)$.

In a similar way we will define the input-decoupling zeros of the positive electrical circuits.

Definition 4.1. Let $\bar{A}_{2}$ be the matrix of unreachable part of the electrical circuit (2.1). The zeros $s_{i 1}, s_{i 2}, \ldots, s_{i \bar{n}_{2}}$ of the characteristic polynomial

$$
\operatorname{det}\left[I_{\bar{n}_{2}} s-\bar{A}_{2}\right]=s^{\bar{n}_{2}}+\bar{a}_{\bar{n}_{2}-1} s^{\bar{n}_{2}-1}+\ldots+\bar{a}_{1} s+\bar{a}_{0}
$$

of the matrix $\bar{A}_{2}$ are called the input-decoupling zero of the positive system (2.1). The list of the input-decoupling zeros will be denoted by $Z_{i}=\left\{s_{i 1}, s_{i 2}, \ldots, s_{i n_{2}}\right\}$. 
Theorem 4.1. The state vector $x(t)$ of the positive electrical circuit (2.1) is independent of the input-decoupling zeros for any input $u(t)$ and zero initial conditions.

Proof. From (2.1) for zero initial conditions $x(0)=0$ we have

$$
X(s)=\operatorname{det}\left[I_{n} s-A\right]^{-1} B U(s),
$$

where $X(s)$ and $U(s)$ are Laplace transforms of $x(t)$ and $u(t)$, respectively. Taking into account (3.2) we obtain

$$
\begin{aligned}
& X(s)=\left[I_{n} s-P^{-1} \bar{A} P\right]^{-1} P^{-1} B U(s)=P^{-1}\left[I_{n} s-\bar{A}\right]^{-1} B U(s) \\
& \quad=P^{-1}\left[\begin{array}{cc}
I_{\bar{n}_{1}} s-\bar{A}_{1} & 0 \\
0 & I_{\bar{n}_{2}} s-\bar{A}_{2}
\end{array}\right]^{-1}\left[\begin{array}{c}
\bar{B}_{1} \\
0
\end{array}\right] U(s)=P^{-1}\left[\begin{array}{c}
{\left[I_{\bar{n}_{1}} s-\bar{A}_{1}\right]^{-1} \bar{B}_{1}} \\
0
\end{array}\right] U(s) .
\end{aligned}
$$

From (4.3) it follows that $X(s)$ is independent of the matrix $\bar{A}_{2}$ and of the input-decoupling zeros for any input $u(t)$.

Example 4.1. (continuation of Example 3.1) In Example 3.1 it was shown that for the unreachable pair $\left(\bar{A}_{2}, \bar{B}_{2}=0\right)$ the matrix $\bar{A}_{2}$ has the form

$$
\bar{A}_{2}=\left[-\frac{R_{2}}{L_{2}}\right] .
$$

Therefore, by Definition 4.1 the electrical circuit shown in Figure 3.1 has one input-decoupling zero

$$
s_{i 1}=-\frac{R_{2}}{L_{2}} .
$$

Note that the input-decoupling zero corresponds to the mesh without the source voltage $\left(e_{2}=0\right)$.

For standard continuous-time linear systems the output-decoupling zeros are defined as the eigenvalues of the matrix of the unobservable part of the system. In a similar way we will define the output-decoupling zeros of the positive electrical circuits.

Definition 4.2. Let $\hat{A}_{2}$ be the matrix of unobservable part of the electrical circuit (2.15). The zeros $s_{O 1}, s_{O 2}, \ldots, s_{o \hat{n}_{2}}$ of the characteristic polynomial

$$
\operatorname{det}\left[I_{\hat{n}_{2}} s-\hat{A}_{2}\right]=s^{\hat{n}_{2}}+\hat{a}_{\hat{n}_{2}-1} s^{\hat{n}_{2}-1}+\ldots+\hat{a}_{1} s+\hat{a}_{0}
$$

of the matrix $\hat{A}_{2}$ are called the output-decoupling zero of the positive electrical circuit (2.15). The list of the output-decoupling zeros will be denoted by $Z_{o}=\left\{s_{o 1}, s_{o 2}, \ldots, s_{o \hat{n}_{2}}\right\}$.

Theorem 4.1. The output vector $y(t)$ of the positive electrical circuit (2.15) is independent of the output-decoupling zeros for any input $\bar{u}(t)=B u(t)$ and zero initial conditions.

Proof is similar to the proof of Theorem 4.1.

Example 4.2. (continuation of Example 3.2) In Example 3.2 it was shown that the matrix $\hat{A}_{2}$ of the unobservable pair has the form 


$$
\hat{A}_{2}=\left[\begin{array}{cc}
-\frac{R_{1}}{L_{1}} & 0 \\
0 & -\frac{R_{2}}{L_{2}}
\end{array}\right] .
$$

Therefore, by Definition 4.2 the positive electrical circuit shown in Figure 3.1 has two outputdecoupling zero

$$
s_{o 1}=-\frac{R_{1}}{L_{1}}, s_{o 2}=-\frac{R_{2}}{L_{2}} .
$$

Following the same way as for standard continuous-time linear systems we define the inputoutput decoupling zeros of the positive systems as follows.

Definition 4.3. Zeros $s_{i o}^{(1)}, s_{i o}^{(2)}, \ldots, s_{i o}^{(k)}$ which are simultaneously the input-decoupling zeros and the output-decoupling zeros of the positive electrical circuit are called the input-output decoupling zeros of the positive electrical circuit, i.e.

$$
s_{i o}^{(j)} \in Z_{i} \text { and } s_{i o}^{(j)} \in Z_{o} \text { for } j=1,2, \ldots, k ; k \leq \min \left(\bar{n}_{2}, \hat{n}_{2}\right) .
$$

The list of input-output decoupling zeros will be denoted by $Z_{i o}=\left\{z_{i o}^{(1)}, z_{i o}^{(2)}, \ldots, z_{i o}^{(k)}\right\}$.

Example 4.3. Consider the positive electrical circuit shown in Figure 3.1 with the matrices $A$, $B, C$ given by (3.4b) and (3.12). In Example 4.1 it was shown that the electrical circuit has one input-decoupling zero $s_{i 1}=-R_{2} / L_{2}$ and in Example 4.2 that the electrical circuit has two output-decoupling zeros $s_{o 1}=-R_{1} / L_{1}, s_{o 2}=-R_{2} / L_{2}$. Therefore, by Definition 4.3 the positive electrical circuit has one input-output decoupling zero $s_{i o}^{(1)}=-R_{2} / L_{2}$.

\section{Concluding remarks}

New necessary and sufficient conditions for the reachability and observability of the positive linear electrical circuits have been established. The definitions of the input-decoupling zeros, output-decoupling zeros and input-output decoupling zeros of the positive electrical circuits have been proposed. Some properties of the new decoupling zeros have been discussed. The considerations have been illustrated by numerical examples of positive electrical circuits (systems) composed of resistors, coils and voltage source. An open problem is an extension of these considerations to fractional discrete-time and continuous-time positive linear systems and fractional electrical circuits.

\section{Acknowledgment}

This work was supported under work $\mathrm{S} / \mathrm{WE} / 1 / 11$. 


\section{References}

[1] Antsaklis P.J., Michel A.N., Linear Systems. Birkhauser, Boston (2006).

[2] Farina L., Rinaldi, S. Positive Linear Systems; Theory and Applications. J. Wiley, New York (2000).

[3] Kaczorek T., Positive 1D and 2D systems. Springer Verlag, London (2001).

[4] Kaczorek T., Linear Control Systems. Vol. 1, J. Wiley, New York 1993.

[5] Kaczorek T., Decomposition of the pairs $(A, B)$ and $(A, C)$ of the positive discrete-time linear systems. Archives of Control Sciences 20(3): 341-361 (2010).

[6] Kaczorek T., Decoupling zeros of positive discrete-time linear systems. Circuit and Systems 1: 41-48 (2010).

[7] Kaczorek T., Positivity and reachability of fractional electrical circuits. Acta Mechanicata et Automatica 5(2): 42-51 (2011).

[8] Kailath T., Linear Systems. Prentice-Hall, Englewood Cliffs, New York (1980).

[9] Kalman R.E., Mathematical Descriptions of Linear Systems. SIAM J. Control 1: 152-192 (1963).

[10] Kalman R.E., On the General Theory of Control Systems. Proc. Of the First Intern. Congress on Automatic Control, Butterworth, London, pp. 481-493 (1960).

[11] Rosenbrock H.H., Comments on poles and zeros of linear multivariable systems: a survey of the algebraic geometric and complex variable theory. Intern. J. Control. 26(1): 157-161 (1977).

[12] Rosenbrock H.H., State-Space and Multivariable Theory. J. Wiley, New York (1970).

[13] Tokarzewski J., Finite zeros of positive linear discrete-time systems. Bull. Pol. Acad. Sci. Tech. 59(3): 287-292 (2011).

[14] Tokarzewski J., Finite zeros of positive continuous-time systems. Bull. Pol. Acad. Sci. Tech. 59(3): 293-298 (2011).

[15] Tokarzewski J., Finite Zeros in Discrete-Time Control Systems. Springer-Verlag, Berlin (2006).

[16] Wolovich W.A., Linear Multivariable Systems, Springer-Verlag New York (1974).

[17] Valcher M.E., On the initial stability and asymptotic behavior of $2 D$ positive systems, IEEE Trans. on Circuits and Systems - I, 44(7): 602-613 (1977). 